\title{
Analyticity Properties of Production Amplitudes
}

\author{
R. Ascoli* AND A. Minguzzi \\ CERN, Geneva, Switzerland \\ (Received December 28, 1959)
}

\begin{abstract}
We study the analytic properties of production amplitudes as functions of the momentum transfer $\Delta^{2}$ between one of the incoming particles and one of the outgoing particles, when the total energy and the three further parameters determining the relative motion of the three outgoing particles in the center-of-mass system are held fixed. We find that suitable combinations of the amplitudes are analytic functions of $\Delta^{2}$ regular within an ellipse in the $\Delta^{2}$ plane. It is also shown that in the same domain the cross section $\partial^{2} \sigma / \partial \Delta^{2} \partial w^{2}$ is an analytic and regular function of $\Delta^{2}, w^{2}$ being the total mass of two of the outgoing particles. The poles in $\Delta^{2}$ conjectured by Chew and Low never lie inside the domain of regularity.
\end{abstract}

\section{INTRODUCTION}

$T^{\mathrm{H}}$ HE purpose of the present work is to investigate whether production amplitudes and cross sections have analytic properties, as functions of momentum transfer, analogous to those found by Lehmann ${ }^{1}$ for scattering amplitudes. Such a problem is particularly interesting in connection with the extrapolation method proposed by Chew and Low $^{2}$ to derive scattering cross sections from suitable production experiments.

\section{ANALYTICITY PROPERTIES OF PRODUCTION AMPLITUDES}

We consider a process where a charged particle of mass $m$ (nucleon) with momentum $p$ collides with a neutral particle of mass $\mu$ (meson) with momentum $k$ producing a charged particle of mass $m^{\prime}$ with momentum $p^{\prime}$ and two neutral particles of masses $\mu^{\prime}, \mu^{\prime \prime}$ and with momenta $k^{\prime}$ and $k^{\prime \prime}$, respectively. We disregard spins.

A process of this kind will depend on 5 invariants. It is convenient to choose for such parameters the total energy in the c.m. system $W^{2}=(p+k)^{2}$, the momentum transfer of two particles (we choose for definiteness the one between the two charged particles) $\Delta^{2}=-\left(p-p^{\prime}\right)^{2} / 4$, and the mass $w^{2}=\left(k^{\prime}+k^{\prime \prime}\right)^{2}$ of the system of the two final neutral particles. Further we use two parameters determining the direction of the vector $\mathbf{k}^{\prime}-\mathbf{k}^{\prime \prime}$ in the c.m. system.

We define the $T$ matrix

$$
\left.\left\langle p^{\prime} k^{\prime} k^{\prime \prime} \text { out }\right| p k \text { in }\right\rangle=i \delta^{(4)}\left(p+k-p^{\prime}-k^{\prime}-k^{\prime \prime}\right) T,
$$

and $T$ as usual is given by

$$
\begin{aligned}
& T=-\int d^{4} x e^{-\frac{1}{2} i(k-p) x} \\
& \quad \times\left\langle p^{\prime} k^{\prime} k^{\prime \prime} \text { out }\left|R^{\prime} A(-x / 2) \psi(x / 2)\right| 0\right\rangle,
\end{aligned}
$$

where

$$
\begin{aligned}
R^{\prime} A(x) \psi(y) \equiv-i\left(\square_{x}-\mu^{2}\right) & \left(\square_{y}-m^{2}\right) \\
& \times \theta(x-y)[A(x), \psi(y)],
\end{aligned}
$$

and $A(x)$ and $\psi(x)$ are the field operators of the initial particles.

\footnotetext{
* On leave of absence from the Institute of Physics of the University of Torino, Torino, Italy.

${ }^{1}$ H. Lehmann, Nuovo cimento 10, 579 (1958)

2 G. F. Chew and F. E. Low, Phys. Rev. 115, 1640 (1959).
}

At this point we make use of the spectral condition and the local commutability. This we do by applying the Dyson ${ }^{3}$ representation to the matrix element of formula (2).

We obtain

$$
T=\int d^{4} u \int d \kappa^{2} \frac{\varphi\left(\kappa^{2}, u, p^{\prime}, k^{\prime}, k^{\prime \prime}\right)}{\left[\frac{1}{2}(k-p)-u\right]^{2}-\kappa^{2}},
$$

where the support of the $\varphi$ function, owing to momentum energy conservation, is defined by the conditions that both the vectors $\frac{1}{2}(p+k)+u$ and $\frac{1}{2}(p+k)-u$ lie in the forward light cone and

$$
\begin{aligned}
& \kappa \geqq \max \left\{0, m_{1}-\left[\left(\frac{p+k}{2}+u\right)^{2}\right]^{\frac{1}{2}},\right. \\
&\left.m_{2}-\left[\left(\frac{p+k}{2}-u\right)^{2}\right]^{\frac{1}{2}}\right\},
\end{aligned}
$$

where $m_{1}$ and $m_{2}$ are the masses of the lowest intermediate states which contribute to the two terms of the unretarded commutator. $\varphi$ is a relativistically invariant function of $\kappa^{2}$ and of the four four-vectors $u, p^{\prime}, k^{\prime}, k^{\prime \prime}$. Alternatively, it may be considered as a function of $\kappa^{2}$, $u, p^{\prime}+k^{\prime}+k^{\prime \prime}, k^{\prime}+k^{\prime \prime}, k^{\prime}-k^{\prime \prime}$.

Here it is convenient to introduce in the center mass system, a polar coordinate system in three-dimensional space with the polar axis perpendicular to the plane defined by the vectors $\mathbf{p}$ and $\mathbf{p}^{\prime}$; the azimuths are counted starting from the direction of $\mathbf{p}^{\prime}$. In this system we call $\alpha, \beta$ the polar coordinates of $\mathbf{u}$ and $a, b$ the polar coordinates of $\mathbf{k}_{1}-\mathbf{k}_{2}$. The polar coordinates of $\mathbf{p}$ are $\theta, \pi / 2$. The choice of this reference is suitable because $\varphi$ will then not depend on $\theta$.

In this system it turns out that $\varphi$ depends on $\kappa^{2}, u_{0}$, $u^{2}, W, w, k_{0}^{\prime}, k_{0}^{\prime \prime}, \mathbf{u} \cdot\left(\mathbf{k}^{\prime}-\mathbf{k}^{\prime \prime}\right), \mathbf{u} \cdot \mathbf{p}^{\prime}$.

The quantities $k_{0}^{\prime}, k_{0}^{\prime \prime},\left|\mathbf{k}^{\prime}-\mathbf{k}^{\prime \prime}\right|,\left|\mathbf{p}^{\prime}\right|$ may be expressed through $W, w, \sin b \cos a$. Indeed $\left|\mathbf{p}^{\prime}\right|$ depends only on $W$ and $w$, the quantities $\left|\mathbf{k}^{\prime}\right|,\left|\mathbf{k}^{\prime \prime}\right|,\left|\mathbf{k}^{\prime}-\mathbf{k}^{\prime \prime}\right|$ are solutions of the system of equations:

$$
\begin{aligned}
\left(\left|\mathbf{k}^{\prime}\right|^{2}+\mu^{\prime 2}\right)^{\frac{1}{2}}+\left(\left|\mathbf{k}^{\prime \prime}\right|^{2}+\mu^{\prime \prime 2}\right)^{\frac{1}{2}} & =\left(w^{2}+\left|\mathbf{p}^{\prime}\right|^{2}\right)^{\frac{1}{2}}, \\
2\left(\left|\mathbf{k}^{\prime}\right|^{2}+\left|\mathbf{k}^{\prime \prime}\right|^{2}\right) & =\left|\mathbf{p}^{\prime}\right|^{2}+\left|\mathbf{k}^{\prime}-\mathbf{k}^{\prime \prime}\right|^{2}, \\
\left|\mathbf{k}^{\prime}\right|^{2}-\left|\mathbf{k}^{\prime \prime}\right|^{2} & =-\left|\mathbf{p}^{\prime}\right|\left|\mathbf{k}^{\prime}-\mathbf{k}^{\prime \prime}\right| \sin b \cos a .
\end{aligned}
$$

${ }^{3}$ F. J. Dyson, Phys. Rev. 110, 1460 (1958). 
Thus $\varphi$ is function of $\kappa^{2}, u_{0}, u^{2}, W, w, \sin b \cos a, \sin \beta \cos \alpha, \cos \beta \cos b-\sin \beta \sin b \cos (\alpha-a)$. Consequently we may write, analogously to Lehmann:

with

$$
T(W, w, \cos \theta, a, b)=\int_{x_{0}(W)}^{\infty} d x \int^{2 \pi} d \alpha \frac{\bar{\varphi}\left(\kappa^{2}, W, w, b, \cos a, \cos \alpha, \cos (\alpha-a)\right)}{x-\cos (\theta-\alpha)},
$$

$\bar{\varphi}\left(\kappa^{2}, W, w, b, \cos a, \cos \alpha, \cos (\alpha-a)\right)$

$$
\begin{array}{r}
=-\frac{1}{4 \pi K} \int d u_{0} \int u d u \int d \kappa^{2} \int_{0}^{\pi} d \beta \delta\left(x-\frac{K^{2}+u^{2}+\kappa^{2}-\left[u_{0}+\left(m^{2}-\mu^{2}\right) / 2 W\right]^{2}}{2 K u \sin \beta}\right) \\
\times \varphi\left(\kappa^{2}, u_{0}, u^{2}, W, w, b, \cos a, \cos \alpha, \cos (\alpha-a)\right),
\end{array}
$$

where $K$ is the momentum of the incident particles in the center-of-mass system and $x_{0}(W)$ is the lower value of the variable $x$ when $u$ and $\kappa^{2}$ vary within the support of the $\varphi$ function. It is:

$$
x_{0}(W)=\left(1+\frac{\left(m_{1}^{2}-\mu^{2}\right)\left(m_{2}^{2}-m^{2}\right)}{K^{2}\left[W^{2}-\left(m_{1}-m_{2}\right)^{2}\right]}\right)^{\frac{1}{2}} .
$$

Let us consider the combinations

$$
T^{+}=T(W, w, \cos \theta, a, b)+T(W, w, \cos \theta, 2 \pi-a, b),
$$

and

$T^{+}$may be written:

$$
T^{-}=T(W, w, \cos \theta, a, b)-T(W, w, \cos \theta, 2 \pi-a, b)
$$

$$
\begin{aligned}
T^{+}=\int_{x_{0}(W)}^{\infty} d x \int_{0}^{\pi} d \alpha \frac{\bar{\varphi}(\cos a, \cos \alpha, \cos (\alpha-a))}{x-\cos (\theta-\alpha)}+\frac{\bar{\varphi}(\cos a, \cos \alpha, \cos (\alpha+a))}{x-\cos (\theta-\alpha)} & +\frac{\bar{\varphi}(\cos a, \cos \alpha, \cos (\alpha+a))}{x-\cos (\theta+\alpha)}+\frac{\bar{\varphi}(\cos a, \cos \alpha, \cos (\alpha-a))}{x-\cos (\theta+\alpha)}
\end{aligned}
$$

where we have explicitly written in $\bar{\varphi}$ only the variables which we are interested in.

We have

$$
T^{+}=\int_{x_{0}(W)}^{\infty} d x \int_{0}^{\pi} d \alpha \frac{2(x-\cos \theta \cos \alpha)[\bar{\varphi}(\cos a, \cos \dot{\alpha}, \cos (\alpha-a))+\bar{\varphi}(\cos a, \cos \alpha, \cos (\alpha+a))]}{(x-\cos \theta \cos \alpha)^{2}-\left(1-\cos ^{2} \theta\right) \sin ^{2} \alpha}
$$

In the same way:

$$
\frac{T^{-}}{\sin \theta}=\int_{x_{0}(W)}^{\infty} d x \int_{0}^{\pi} d \alpha \frac{2 \sin \alpha[\bar{\varphi}(\cos a, \cos \alpha, \cos (\alpha-a))-\bar{\varphi}(\cos a, \cos \alpha, \cos (\alpha+a))]}{(x-\cos \theta \cos \alpha)^{2}-\left(1-\cos ^{2} \theta\right) \sin ^{2} \alpha}
$$

So we see that the integrands appearing in these expressions of $T^{+}$and $T^{-} / \sin \theta$ are analytic functions of $\cos \theta$ regular as long as the denominator does not vanish. Therefore the integrals are analytic and regular functions of $\cos \theta$ at least in the region for which the denominator does not vanish in the domain of integration. This regularity region is, as in the case of scattering treated by Lehmann, an ellipse with focii at the points -1 and +1 and semiaxes $x_{0}$ and $\left(x_{0}^{2}-1\right)^{\frac{1}{2}}$. This result holds also separately for the real and the imaginary parts of $T^{+}$and $T^{-} / \sin \theta$.

From the analyticity properties of $T^{+}$and $T^{-} / \sin \theta$ it follows that also

$$
\begin{aligned}
\left|T^{+}\right|^{2}+\left|T^{-}\right|^{2}=2[ & |T(W, w, \cos \theta, a, b)|^{2} \\
& \left.+|T(W, w, \cos \theta, 2 \pi-a, b)|^{2}\right]
\end{aligned}
$$

is an analytic function of $\cos \theta$ in the same ellipse. So in contrast to the case of elastic scattering considered by Lehmann, it has not been possible to conclude that $T$ itself is an analytic function of $\cos \theta$, due to the fact that $\varphi$ does not depend only on $\cos \alpha$. Indeed in the case of elastic scattering only one three-dimensional scalar product is present in $\varphi$, whereas in the production amplitude three three-dimensional scalar products 
occur. So we cannot exclude the presence of a cut of $T$ in the $\cos \theta$ plane due to the presence of odd powers of $\sin \theta$ in the integrand of (7). ${ }^{4}$

However, the particular sum of squared amplitudes appearing in (12) is analytic in $\cos \theta$ within the ellipse.

\section{ANALYTICITY PROPERTIES OF PRODUCTION CROSS SECTIONS}

It is immediate to show that also $\partial^{2} \sigma / \partial w^{2} \partial \Delta^{2}$ where $\sigma$ is the production cross section, is analytic and regular in the same domain. In fact we have

$$
\begin{array}{r}
\sigma=\frac{\pi^{2}}{\left[(k p)^{2}-m^{2} \mu^{2}\right]^{\frac{1}{2}}} \int|T|^{2} \delta^{(4)}\left(k^{\prime}+k^{\prime \prime}+p^{\prime}-k-p\right) \delta\left(k^{\prime 2}-\mu^{\prime 2}\right) \delta\left(k^{\prime \prime 2}-\mu^{\prime \prime 2}\right) \\
\quad \times \delta\left(p^{\prime 2}-m^{\prime 2}\right) \theta\left(k^{\prime}\right) \theta\left(k^{\prime \prime}\right) \theta\left(p^{\prime}\right) d^{4} k^{\prime} d^{4} k^{\prime \prime} d^{4} p^{\prime} .
\end{array}
$$

After integration over $k_{0}{ }^{\prime}, k_{0}{ }^{\prime \prime}, p_{0}{ }^{\prime}$ we introduce the variables $\mathbf{k}^{\prime}+\mathbf{k}^{\prime \prime}, \mathbf{k}^{\prime}-\mathbf{k}^{\prime \prime}=\mathbf{q}$ and integrate over the first one; then we have in the center-of-mass system

$$
\sigma=\frac{\pi^{2}}{64\left[(p k)^{2}-m^{2} \mu^{2}\right]^{\frac{1}{2}}} \int \frac{|T|^{2}}{p_{0}{ }^{\prime} k_{0}{ }^{\prime} k_{0}{ }^{\prime \prime}} \delta\left[W-\left(\left|\mathbf{k}^{\prime}\right|^{2}+\mu^{\prime 2}\right)^{\frac{1}{2}}-\left(\left|\mathbf{k}^{\prime \prime}\right|^{2}+\mu^{\prime \prime 2}\right)^{\frac{1}{2}}-\left(\left|\mathbf{p}^{\prime}\right|^{2}+m\right)^{\frac{1}{2}}\right] d^{3} q d^{3} p^{\prime} .
$$

The integration over $|\mathbf{q}|$ enables us to eliminate the $\delta$ function of energy with the result

$$
\sigma=\frac{\pi^{3}}{32\left[(p k)^{2}-m^{2} \mu^{2}\right]^{\frac{1}{2}}} \int \frac{|T|^{2}}{p_{0}{ }^{\prime} k_{0}{ }^{\prime} k_{0}{ }^{\prime \prime}} d \cos \theta\left|\mathbf{p}^{\prime}\right|^{2} \frac{d\left|\mathbf{p}^{\prime}\right|}{d w^{2}}|\mathbf{q}|^{2} \frac{d|\mathbf{q}|}{d W} d \cos b d a d w^{2},
$$

where it is understood that the derivative $d|\mathbf{q}| / d W$ is done at fixed $w, \cos \theta, a, b$; the derivative $d\left|\mathbf{p}^{\prime}\right| / d w^{2}$ is done at fixed $W$ and $T^{2}, p_{0}{ }^{\prime}, k_{0}{ }^{\prime}, k_{0}{ }^{\prime \prime},\left|\mathbf{p}^{\prime}\right|^{2}, d\left|\mathbf{p}^{\prime}\right| / d w^{2},|\mathbf{q}|^{2}, d|\mathbf{q}| / d W$ are expressed in terms of $W, \cos \theta, w, a, b$.

Now the quantities $p_{0}^{\prime},\left|\mathbf{p}^{\prime}\right|^{2}, d\left|\mathbf{p}^{\prime}\right| / d w^{2}$ are independent on $a$. Moreover, owing to (6) the quantities $k_{0}{ }^{\prime}, k_{0}{ }^{\prime \prime}$, $|\mathbf{q}|^{2}, d|\mathbf{q}| / d W$ depend upon $a$ only through $\cos a$. Therefore the integral in (15) can be written:

$$
\sigma=\frac{\pi^{3}}{64\left[(p k)^{2}-m^{2} \mu^{2}\right]^{\frac{1}{2}}} \int d \cos \theta\left|\mathbf{p}^{\prime}\right|^{2} \frac{d\left|\mathbf{p}^{\prime}\right|}{d w^{2}} d w^{2} \frac{1}{p_{0}{ }^{\prime}} \int \frac{|T(a)|^{2}+|T(2 \pi-a)|^{2}}{k_{0}{ }^{\prime} k_{0}{ }^{\prime \prime}}|\mathbf{q}|^{2} \frac{d|\mathbf{q}|}{d W} d \cos b d a,
$$

from which we have:

$$
\frac{\partial^{2} \sigma}{\partial w^{2} \partial(\cos \theta)}=\frac{\pi^{3}}{64\left[(p k)^{2}-m^{2} \mu^{2}\right]^{\frac{1}{2}}}\left|\mathbf{p}^{\prime}\right|^{2} \frac{d\left|\mathbf{p}^{\prime}\right|}{d w^{2}} \frac{1}{p_{0}{ }^{\prime}} \int \frac{|T(a)|^{2}+|T(2 \pi-a)|^{2}}{k_{0}{ }^{\prime} k_{0}{ }^{\prime \prime}}|\mathbf{q}|^{2} \frac{d|\mathbf{q}|}{d W} d \cos b d a .
$$

Now we have proved that the quantity $|T(a)|^{2}$ $+|T(2 \pi-a)|^{2}$ is an analytic function of $\cos \theta$ regular in an ellipse. On the other hand, all the remaining quantities in (17) are independent on $\cos \theta$, so $\partial^{2} \sigma / \partial w^{2} \partial \cos \theta$ is also an analytic function of $\cos \theta$ in the same region.

By integration of (17) over $w^{2}$ in the allowed space we find that $\partial \sigma / \partial \cos \theta$ is also an analytic function of $\cos \theta$ regular at least in the same region.

From (17) we also immediately find that $\partial^{2} \sigma / \partial w^{2} \partial \Delta^{2}$ is an analytic function of $\Delta^{2}$ regular in a region of the $\Delta^{2}$ plane which is the mapping of the ellipse in the $\cos \theta$ plane.

\section{DISCUSSION}

Let us now compare this result with the conjecture of Chew and Low on the analytic properties of $\partial^{2} \sigma / \partial w^{2} \partial \Delta^{2}$ considered as a function of $\Delta^{2}$ at fixed $W$ and $w$.

\footnotetext{
${ }^{4}$ This result may depend on the choice of the parameters. One cannot exclude that another choice of variables (for instance $W^{2}$, $\Delta^{2}, w^{2}, k^{\prime} k, k^{\prime \prime} k$ ) allows one to deduce analyticity properties of the production amplitude (for instance, as function of $\Delta^{2}$ at fixed $\left.W^{2}, w^{2}, k^{\prime} k, k^{\prime \prime} k\right)$ analogous to those holding for the elastic amplitude. However, it seems that the variables we have chosen are the only ones which enable to deduce analyticity properties using only the Dyson representation.
}

In the case of elastic scattering, for instance $N+N$ $\rightarrow N+N$, one expects a pole due to the exchange of one meson. The comparison with the results of Lehmann shows that this pole never lies inside the ellipse and reaches one of the intersections of the ellipse with the real axis for one energy value.

For production processes we have to distinguish two cases:

(1) A pole in the $\Delta^{2}$ variable due to the exchange of one particle is expected in both the production process and in the elastic process with the same initial particles: for instance, $N+N \rightarrow N+N$ and $N+N \rightarrow N+N+\pi$.

(2) Owing to some selection rule this is not the case; as for instance in the processes $N+\pi \rightarrow N+\pi$ and $N+\pi \rightarrow N+\pi+\pi$.

Then it may be proved that for the production processes of type (1) the pole in $\cos \theta$ in the production cross section is farther from the physical range of $\cos \theta$ than the pole of the corresponding elastic cross section. So, as long as it has been proved that the expected pole in the elastic process never lies inside the ellipse, it follows 
that the expected pole in the production process always lies outside the ellipse.

Further it can be shown that in any case in the production processes the expected pole attains its minimum distance from the physical range when $w^{2}$ has the minimum value $\left(\mu^{\prime}+\mu^{\prime \prime}\right)^{2} .^{5}$

We have investigated the cases of type (2), $p+\pi$ $\rightarrow p+\pi+\pi$ and $p+K \rightarrow p+K+\pi$ for the threshold value of $w^{2}$. In both cases we have found that the expected pole never lies inside the ellipse, and only for one value of $W$ reaches the ellipse. This value is $W=m+3.5 \mu$ and $W=M+M_{K}+3.6 \mu$ for the two processes, respectively.

${ }^{5}$ Of course $\partial^{2} \sigma / \partial w^{2} \partial \Delta^{2}$ tends to zero as $w^{2}$ goes to $\left(\mu^{\prime}+\mu^{\prime \prime}\right)^{2}$ this is due to the phase space factor of the final particles of momenta $k^{\prime}$ and $k^{\prime \prime}$. In this case it is more convenient to consider the quantity

$$
\frac{1}{\left[w^{4}+\left(\mu^{\prime 2}-\mu^{\prime \prime 2}\right)^{2}-2 w^{2}\left(\mu^{\prime 2}+\mu^{\prime \prime 2}\right)\right]^{\frac{1}{3}}} \frac{\partial^{2} \sigma}{\partial w^{2} \partial \Delta^{2}}
$$

which in general is different from zero at $w^{2}=\left(\mu^{\prime}+\mu^{\prime \prime}\right)^{2}$ and has the same analyticity properties of $\partial^{2} \sigma / \partial w^{2} \partial \Delta^{2}$ as function of $\Delta^{2}$.

\section{CONCLUSIONS}

We may summarize the results as follows. It has not been possible to prove an analytic property of the production amplitude analogous to the one holding for the elastic amplitudes as function of $\cos \theta$. However, we have shown that some special combinations of the amplitudes [formulas (8)] are analytic within the same region of the $\cos \theta$ plane found by Lehmann in the case of elastic scattering with the same incoming particles.

Finally, we have proved that the conjectures of Chew and Low which refer to the analyticity properties of the cross section (17) as a function of $\cos \theta$ are confirmed at least within the Lehmann ellipse.

\section{ACKNOWLEDGMENTS}

We would like to thank Professor C. J. Bakker and Professor M. Fierz for hospitality at the CERN Theoretical Study Division. Furthermore, one of us, (R.A.), would like to thank the "Fondazione Francesco Somaini" for financial support.

\title{
Calculation of Single-Particle Energies in the Theory of Nuclear Matter
}

\author{
Keith A. Brueckner \\ University of California, La Jolla, California \\ John L. GAMmeL \\ Los Alamos Scientific Laboratory, \\ University of California, Los Alamos, New Mexico \\ AND \\ Joseph T. KuBIS \\ Princeton University, Princeton, New Jersey \\ (Received December 28, 1959)
}

\begin{abstract}
The rearrangement energy corrections to the single-particle energies have been evaluated, using the procedure of Brueckner and Goldman. The shift is shown to be due largely to the second-and third-order rearrangement energy diagrams, the corrected energy at the Fermi surface now nearly agreeing with the mean binding energy.

The change of the single-particle energies of virtual excitations due to rearrangement effects is also determined and shown to shift the mean binding energy by $1.5 \mathrm{Mev}$.
\end{abstract}

\section{INTRODUCTION}

T has been shown by Brueckner and Goldman ${ }^{1}$ that 1 a redefinition of single-particle energy within the framework of the $K$-matrix approximation ${ }^{2}$ for the total energy of a Fermion many-body system leads to singleparticle energies which satisfy the separation energy theorem of Hugenholtz and Van Hove. ${ }^{3}$ It is the purpose of this paper to give the quantitative results obtained

\footnotetext{
${ }^{1}$ K. A. Brueckner and D. T. Goldman, Phys. Rev. 117, 207 (1960); hereafter referred to as BG.

${ }^{2}$ K. A. Brueckner, Phys. Rev. 100, 36 (1955).

${ }^{3}$ N. M. Hugenholtz and L. Van Hove, Physica 24, 363 (1958).
}

for the single-particle energies and also the ground-state energies from an application of the $B G$ procedure.

\section{SINGLE-PARTICLE ENERGY}

Following BG, we start from the $K$-matrix expression for the ground-state energy, considered as a function of the occupation numbers of the Fermi gas, i.e.,

$$
E\left(n_{\alpha}\right)=\sum_{i} n_{i} \frac{p_{i}{ }^{2}}{2 M}+\frac{1}{2} \sum_{i j} n_{i} n_{j}\left(K_{i j, i j}-K_{i j, j i}\right),
$$

